\title{
Highlights of ICD-11 Classification of Mental, Behavioral, and Neurodevelopmental Disorders
}

\author{
Anirban Gozi
}

\begin{abstract}
International classification of diseases (ICD)-11 is expected to be operative on the first day of January 2022. The key principle in this revision is simplification of the coding structure, electronic tooling, along with incorporation of advancements that occurred over past 30 years, since the publication of ICD-10. For a classification of mental disorders, development of the ICD-11 has been the most global, multilingual, multidisciplinary, and participative revision process by far. Adoption of a life-span approach, incorporation of a dimensional approach, provision of consistent and systematically characterized information as per recent evidence, and culture-related guidance along with effort for destigmatization are the salient features of this revision. In this review, we highlighted the process of developing the clinical description and diagnostic guidelines $(C D D G)$, discussed the new disorders that were added with rationale, and last described the salient features of disorder grouping, pointing the key changes from ICD-10.

Keywords: Dimensional approaches, ICD-11 classification, International classification of diseases, Life-span approach.

Indian Journal of Private Psychiatry (2019): 10.5005/jp-journals-10067-0030
\end{abstract}

\section{INTRODUCTION}

International classification of diseases is the foundation for the identification of health trends and statistics globally and the international standard for reporting diseases and health conditions. There had been a substantial advancement in medical science over the past 30 years since the endorsement of ICD-10 in May 1990 by the 43rd World Health Assembly-often found to be outdated both clinically and from a classification perspective. Minor updating of the ICD chapters probably would not be sufficient enough. Additionally, increasing need to operate in an electronic environment in this modern era also expedited the development of 11 th version. On June 18, 2018, the World Health Organization (WHO) released a pre-final version of the 11th revision of ICD for mortality and morbidity statistics to its 194 member states, for review and preparation for implementation including translating ICD into their national languages. ${ }^{1}$ International classification of diseases-11 was subjected to be submitted to the 144th Executive Board Meeting on January 2019 and the 72nd World Health Assembly in May 2019; following endorsement, member states will start reporting using ICD-11 on January 1, 2022.

International classification of diseases defines the universe of diseases, disorders, injuries, and other health conditions in a comprehensive, hierarchical fashion that allows for secured storage, retrieval, and analysis of health information for evidence-based decision-making, sharing and comparing health information between hospitals, regions, settings, and countries. Contrary to the prevailing view that clinicians only use the classification to obtain diagnostic codes for administrative and billing purposes, a recent survey ${ }^{2}$ suggests that clinicians somewhat regularly use the CDDG and often review them systematically when making an initial diagnosis. Publication of the CDDG version of ICD-11 is expected following approval of the overall system by the World Health Assembly. ${ }^{3}$

\section{Behind the Lens: Making of ICD-11 CDDG}

The WHO Department of Mental Health and Substance Abuse had been responsible for coordinating the development of major four
Department of Psychiatry, Medical College, Kolkata, West Bengal, India

Corresponding Author: Anirban Gozi, Department of Psychiatry, Medical College, Kolkata, West Bengal, India, Phone: +91 9916740735, e-mail: psygozi@gmail.com

How to cite this article: Gozi A. Highlights of ICD-11 Classification of Mental, Behavioral, and Neurodevelopmental Disorders. Ind J Priv Psychiatry 2019;13(1):11-17.

Source of support: Nil

Conflict of interest: None

ICD-11 chapters: mental, behavioral, and neurodevelopmental disorders; disease of nervous system; sleep-wake disorders; and conditions related to sexual health (jointly with the WHO Department of Reproductive Health and Research).

A series of working groups, generally organized by disorder grouping, were appointed by the WHO Department of Mental Health and Substance Abuse to make the recommendations regarding the structure and content of the ICD-11 CDDG. All groups included representatives from all WHO global regions-Africa, the Americas, Eastern Mediterranean, Western Pacific, Europe, and Southeast Asia-that included almost $80 \%$ of the world's population from lower- and middle-income countries. ${ }^{4}$ Importance of global applicability was also strongly emphasized to working groups. Working groups were asked to submit their recommendations as "content forms," including consistent and systematic information for each disorder that provided the basis for the guidelines and supposed to reduce the lack of consistency often found to be the shortcoming of the ICD-10 CDDG.

There was significant overlapping in time frame and workgroups while the development of the ICD-11 CDDG and the diagnostic and statistical manual-5 (DSM-5) by American Psychiatric Association. ICD-11 working groups were asked to consider the clinical utility and global applicability of material being developed for the DSM-5 aiming to minimize random or arbitrary differences between the 
ICD-11 and the DSM-5, although justified conceptual differences were permitted.

The ICD-11 field study program used novel methodologies for studying the clinical utility of the draft diagnostic guidelines, including their consistency and accuracy of the application by clinicians. Most of the studies have been conducted in a time frame to allow their results to provide a basis for revision of the guidelines to address any observed weaknesses. ${ }^{5}$

The global participation has been ensured by establishing the global clinical practice network (GCPN) to allow directly mental health and primary care professionals from all over the world to participate through internet-based field studies. More than half of GCPN members are physicians, predominantly psychiatrists, and $30 \%$ are psychologists, and it has expanded to include nearly 15,000 clinicians from 155 countries with the gigantic proportions coming from Asia, Europe, and the Americas. ${ }^{6}$ The GCPN studies have been conducted in Japanese, Chinese, Russian, Spanish, and French, in addition to English, and have included an examination of results by language and region to identify potential difficulties in global or cultural applicability. Clinic-based studies have also been conducted through a network of international field study centers located in 14 countries across all WHO global regions conducted in the local language to evaluate the clinical utility and usability of the proposed ICD-11 diagnostic guidelines in natural conditions. ${ }^{7}$

\section{Major Innovations In the ICD-11 CDDG}

The important innovation incorporated in ICD-11 is the "life-span approach," i.e., disorders distributed to the groupings with which they share symptoms rather than onset according to the age, resulting in deletion of the group - disorders usually occurring in childhood and adolescence. For example, separation anxiety disorder was shifted to the anxiety and fear-related disorders grouping.

"Essential features" have been described in ICD-11 that represent the characteristics that a clinician could reasonably expect to find in all cases. Though the lists of essential features in the guidelines superficially resemble diagnostic criteria, arbitrary cutoffs and symptom counts and duration were generally avoided. Such approach ensured the flexible exercise of clinical judgment and increased clinical utility.

Culture-related information was systematically incorporated based on a literature review on cultural influences on psychopathology and its expression for diagnostic grouping as well as a detailed review of culture-related material in the ICD-10 CDDG and the DSM-5.

Another major innovation was incorporating dimensional approaches within an existing categorical system. ${ }^{8,9}$ Dimensional classification focuses on relevant aspects of the clinical presentation, which is more consistent with recovery-based psychiatric rehabilitation approaches. The dimensional potential is robustly realized in the classification of personality disorders. ${ }^{10}$

\section{Overview of Structure of the ICD-11 Chapter on Mental, Behavioral, and Neurodevelopmental Disorders}

The flexible alphanumeric coding structure allowed for a much larger number of groupings than the decimal coding system in ICD-10, which allowed having a maximum of 10 major groupings of disorders. Diagnostic groupings in ICD-11 were created based more closely on scientific evidence and the needs of clinical practice (e.g., anxiety disorders were included as part of the heterogeneous group of neurotic, stress-related, and somatoform disorders). Two formative field studies were conducted ${ }^{11,12}$ to examine the conceptualizations held by mental health professionals around the world regarding the relationships among mental disorders. An effort was made to keep away the disparities between the organizational structures of DSM-5 and ICD-11.

The Kraepelinian organization of disorders followed in ICD-10 was shifted to a more scientific one. The organization principle followed a developmental perspective (neurodevelopmental disorders appear first and neurocognitive disorders last in the classification henceforth) and disorders were grouped together based on putative shared etiological and pathophysiological factors and shared phenomenology as well.

The impractical organic-nonorganic dichotomy for sleepand sex-related disorders was discarded in ICD-11. While a separate chapter has been created for sleep-wake disorders that encompasses all relevant sleep-related diagnoses, another new integrated chapter for conditions related to sexual health has been added to house a unified classification of sexual dysfunctions and sexual pain disorders. ${ }^{13}$

Transgender identity has been destigmatized by moving from the mental disorders chapter to the new sexual health chapter and was renamed as "gender incongruence" explicitly stating that gender variant behavior and preferences alone are not sufficient for making a diagnosis. ${ }^{13}$

\section{Novel Additions In ICD-11}

Quite a few numbers of new disorders have been added to the ICD-11 chapter on mental, behavioral, and neurodevelopmental disorders.

\section{Catatonia}

The change in conceptualization of catatonia had been claimed from many years to give it an individual status, and this is for the first time catatonia was given the status of an independent syndrome. An entirely new diagnostic grouping for catatonia (at the same hierarchical level as anxiety and fear-related disorders, mood disorders, etc.) has been added in the ICD-11. Three out of twelve core features are needed for the diagnosis of this syndrome. Three conditions are included in the new diagnostic grouping: (a) catatonia associated with another mental disorder, (b) catatonia induced by psychoactive substances, including medications, and (c) secondary catatonia, i.e., caused by a medical condition along with the addition of "catatonia not otherwise specified (NOS)" group. ${ }^{14}$

\section{Bipolar Type II Disorder}

DSM-IV has already introduced bipolar type II disorder, requiring an absence of manic episodes along with at least one hypomanic episode plus at least one major depressive episode to diagnose. ICD-11 also included this with the supporting evidence of differences in antidepressant monotherapy response, ${ }^{15}$ neurocognitive measures, ${ }^{15,16}$ genetic effects, ${ }^{17}$ and neuroimaging findings ${ }^{18,19}$ between bipolar I and II disorders.

\section{Body Dysmorphic Disorder}

Recognizing its distinctive symptomatology and similarities to obsessive-compulsive disorder, body dysmorphic disorder has been included in the obsessive-compulsive and related disorder (OCRD) 
grouping in the ICD-11. ${ }^{20}$ Such inclusion excluded the confusing terms like hypochondriasis or dysmorphophobia and made it easy to differentiate from delusional disorders.

\section{Olfactory Reference Disorder}

In clinical settings, such conditions were not uncommon. This disorder is characterized by a persistent preoccupation of emitting a perceived foul or offensive body odor or breath that is either unnoticeable or only slightly noticeable to others. This is accompanied by repeated checking or reassurance-seeking behavior and marked avoidance of social situations. ${ }^{21,22}$ Olfactory reference disorder is included in the ICD-11 OCRD grouping.

\section{Hoarding Disorder}

Individuals affected by hoarding disorder are often underrecognized and undertreated, which argues from a public health perspective for its inclusion in the ICD-11. ${ }^{23}$ Sufficient shreds of evidence support hoarding disorder as a separate and unique disorder. ${ }^{24}$ Clinical characteristics were kept in line of DSM-5, i.e., accumulation of possessions, due to their excessive acquisition or difficulty in discarding them, regardless of their actual value. ${ }^{25}$

\section{Excoriation Disorder}

In view of similarities in phenomenology, patterns of familial aggregation, and putative etiological mechanisms, another new diagnostic subgroup naming body-focused repetitive behavior disorders, has been added to the OCRD grouping, including trichotillomania (was under habit and impulse disorders in ICD-10) and a new condition, excoriation disorder (also known as skin-picking disorder). Excoriation disorder (and trichotillomania) is distinct from other OCRDs, as the behavior found to be rarely preceded by cognitive phenomena such as intrusive thoughts, obsessions, or preoccupations, but sensory experiences. ${ }^{26}$

\section{Complex Post-traumatic Stress Disorder}

The addition of complex post-traumatic stress disorder (PTSD) to the ICD-11 is justified based on the evidence of poorer prognosis and benefit from different treatment modalities as compared to individuals with PTSD42. Complex PTSD describes an additional feature of persistent, pervasive, and enduring disturbances, in affect regulation, self-concept, and relational functioning, and so replaces the overlapping ICD-10 category of enduring personality change after catastrophic experience. ${ }^{27}$

\section{Prolonged Grief Disorder}

In response to the increasing evidence of a distinct and debilitating condition, persisting for an atypically long period following the loss (more than 6 months), that was not adequately described by ICD-10, prolonged grief disorder was added in ICD-11. ${ }^{43}$ Its inclusion and differentiation from the culturally normative bereavement and depressive episode is essential because of the different treatment selection implications and prognoses. ${ }^{28}$

\section{Binge Eating Disorder}

Frequent, recurrent episodes of binge eating (e.g., once a week or more over several months); subjective loss of control; experience of distress, guilt, or disgust; and not regularly followed by inappropriate compensatory behaviors (notable difference from bulimia nervosa) describe this disorder. Weight gain is not an essential feature though might be present. Inclusion of binge eating disorder overlaps ICD-10 diagnoses of other specified or unspecified eating disorder.
Extensive research that has emerged during the last 20 years supports its validity and clinical utility. ${ }^{29}$

\section{Avoidant/restrictive Food Intake Disorder}

Avoidant/restrictive food intake disorder (ARFID) is characterized by intake of an inadequate quantity or variety of food to meet adequate energy or nutritional requirements in the absence of concerns about body weight or shape (difference from anorexia nervosa), resulting in weight loss or failure to gain in childhood or pregnancy. Its inclusion can be considered to be an expansion of the ICD-10 category "feeding disorder of infancy and childhood" and is likely to improve clinical utility across the life span (i.e., ARFID applies to children, adolescents, and adults) as well as maintaining consistency with DSM-5. ${ }^{29,30}$

\section{Body Integrity Dysphoria}

A rare disorder characterized by the persistent desire to have a specific physical disability (e.g., blindness, amputation, paraplegia, deafness) beginning in childhood or early adolescence. Often manifested in fantasizing about having the desired physical disability or engaging in "pretending" behavior. For a few individuals, this desire or preoccupation goes far beyond fantasy, and they pursue actualization of the desire through surgery (i.e., by procuring an elective amputation) or by self-damaging a limb to a degree where amputation remains the only therapeutic option (e.g., freezing a limb in dry ice). ${ }^{31}$

\section{Gaming Disorder}

Gaming disorder has been included in a newly added diagnostic group called "disorders due to addictive behaviors" (which also contains gambling disorder) in view of phenomenological and pathophysiological resemblance that became a global concern. It is differentiated from nonpathological gaming behavior by the production of clinically significant distress or impairment in functioning. ${ }^{32}$

\section{Compulsive Sexual Behavior Disorder}

This is characterized by a persistent pattern of failure to control intense, repetitive sexual impulses or urges for a minimum duration of 6 months or more; repetitive sexual activities become a central focus of the individual's life despite adverse consequences such as repeated relationship disruption even when he or she no longer gets any satisfaction from it.

Although this category phenomenologically resembles behavioral addictions, it is included in the ICD-11 impulse control disorder section in recognition of the lack of definitive information. Such inclusion will help to address unmet needs of treatmentseeking patients and possibly reducing shame and guilt associated with help seeking among distressed individuals. ${ }^{33}$

\section{Intermittent Explosive Disorder}

Although intermittent explosive disorder was introduced in the DSM-III-R, ICD-10 kept it only as an inclusion term under "other habit and impulse disorders." It is reincluded in the ICD-11 impulse control disorders section in recognition of the substantial evidence of its validity and utility in clinical settings. This disorder is characterized by repeated brief episodes of verbal or physical aggression or destruction that represent a failure to control aggressive impulses, out of proportion to the provocation or precipitating psychosocial stressors, diagnosed only if not better explained by another disorders like oppositional defiant disorder, conduct disorder, and bipolar disorder. ${ }^{34}$ 
Highlights of ICD-11 Classification of Mental, Behavioral, and Neurodevelopmental Disorders

\section{Premenstrual Dysphoric Disorder}

In the ICD-11, premenstrual dysphoric disorder (PMDD) is differentiated from a more commonly occurring entity, premenstrual tension syndrome, by the severity. The inclusion of PMDD in the research appendices of the DSM-III-R and DSM-IV stimulated a great deal of research that established its validity and reliability. Premenstrual dysphoric disorder is cross-listed in the subgrouping of depressive disorders due to the prominence of affective symptoms, although its primary location is in the ICD-11 chapter on diseases of the genitourinary system. ${ }^{35}$

\section{Summary of ICD-11 Disorder Grouping AND Modifications}

The addition of new disorders has been elaborated. Here is the summary of changes in the main disorder groupings of the ICD-11 chapter on mental, behavioral, and neurodevelopmental disorders. ICD-11 working groups reviewed the shreds of evidence to achieve clinical utility and global applicability. Field trials were carried out accordingly.

\section{Neurodevelopmental Disorders}

Neurodevelopmental disorders come first in ICD-11 according to the life-span approach followed in entirety. Disorders of acquisition and execution of specific intellectual, language, motor, and social functions have been included here. The notable changes are renaming of mental retardation and disorders of psychological development of ICD-10 to disorders of intellectual development, an applauding effort to decrease the stigma associated. This provides a comprehensive set of behavioral indicators, ${ }^{36}$ tables for intellectual and adaptive functioning domains (conceptual, social, practical), organized according to age groups (early childhood, childhood/ adolescence, and adulthood) and four levels of severity (mild, moderate, severe, profound).

In autism spectrum disorder (ASD), both childhood autism and Asperger's syndrome were incorporated. Qualifiers to embrace the extent of impairment were added.

Attention deficit hyperactivity disorder (ADHD) has been added in this group and replaced the term "hyperkinetic disorder." This was an effort to indicate the common co-occurrence of other neurodevelopmental disorders, developmental onset, as well as to lessen the stigma by differentiating it from dissocial or disruptive disorders. Qualifiers like inattentive and hyperactive-impulsive were retained as ICD-10.

Chronic tic disorders (Tourette syndrome) were actually classified in the chapter on diseases of the nervous system but are cross-listed here in this group.

\section{Schizophrenia and Other Primary Psychotic Disorders}

The term "primary" has been incorporated here to exclude the cases of affective or induced psychosis, ${ }^{37}$ pointing that psychosis being the core feature. Other changes were in accordance with the DSM-5, like deemphasization of Schneiderian first-rank symptoms and elimination of subtypes of schizophrenia due to their lack of predictive validity or utility in treatment selection. Dimensions have been described, including detailed description (positive symptoms/negative symptoms/depressive mood symptoms, manic mood symptoms, psychomotor symptoms/cognitive symptoms). The "polymorphic" picture of the acute and transient psychotic disorder has been particularly emphasized for the diagnosis. Otherwise, no major conceptualization changes were done in schizoaffective disorder or schizotypal disorder.

\section{Mood Disorders}

While diagnosing a mood episode, a longitudinal pattern was given importance in ICD-11. Though symptom count was deemphasized, depressive disorder is one of the few places where it was used. Depressive symptoms are organized into three clusters-affective, cognitive, and neurovegetative. Hopelessness has been given special significance among cognitive symptoms in view of strong evidence of its predictive value. ${ }^{38}$ Persistent mood disorders in ICD-10, consisting of dysthymia and cyclothymia, has been eliminated ${ }^{39}$ and included in depressive disorders and bipolar disorders accordingly. Presence of typical contrapolar mixed symptoms confirms the bipolar type I diagnosis. Several qualifiers were added like mood episodes in partial or in full remission; severity of an episode (mild, moderate, or severe); and qualifiers for melancholic features, prominent anxiety symptoms, presence of panic attacks, seasonal pattern, and rapid cycling. Mixed depressive and anxiety disorder was shifted to depressive disorders in the ICD-11 due to prominence of mood symptoms.

\section{Anxiety and Fear-related Disorders}

Disorders primarily presenting with anxiety or fear were regrouped here. ${ }^{40}$ Separation anxiety disorder and selective mutism were shifted here according to the life-span approach. The ICD-10 hierarchical exclusion rules have been eliminated, as this interfere with specific clinical attention. So generalized anxiety disorder (GAD) can co-occur with depressive disorders as long as symptoms are present independent of mood episodes. In agoraphobia, conceptual broadening is done to discard the narrower concept of fear of open spaces and related situations, such as crowds; rather, ICD-11 gives more stress on specific negative outcomes that would be incapacitating or embarrassing in the situations. Concept of panic disorder is kept same as ICD-10, while a "with panic attacks" qualifier can be applied to the other anxiety disorder diagnosis. And above all, separation anxiety disorder can be diagnosed in adults, where it is most commonly focused on a romantic partner or a child. ${ }^{40}$

\section{OCRDs}

Despite the phenomenological overlap, the OCRD group was introduced for differentiating from other anxiety disorders on the basis of presence of core feature of unwanted thoughts and related repetitive behaviors along with evidence of the shared validators among included disorders from imaging, genetic, and neurochemical studies. ${ }^{20}$ The group includes body dysmorphic disorder, olfactory reference disorder, hypochondriasis (illness anxiety disorder), and hoarding disorder. The subgroup of bodyfocused repetitive behavior disorders, including trichotillomania (hair-pulling disorder) and excoriation (skin-picking) disorder, was introduced as both share the core feature of repetitive behavior without the cognitive aspect of other OCRDs. Tourette syndrome is cross-listed in this group because of its frequent co-occurrence with obsessive compulsive disorder (OCD). International classification of diseases-10 subtypes of OCD are eliminated because of the frequent presence of covert compulsions along with the addition of the qualifier "with poor to absent insight." Depressive disorders are not prohibited anymore to be diagnosed with OCD in view of high co-occurrence. 


\section{Disorders Specifically Associated with Stress}

Reactions to severe stress and adjustment disorders in ICD-10 have been replaced by "disorders specifically associated with stress" in ICD-11. Reactive and disinhibited attachment disorders of childhood are reclassified to this group owing to the life-span approach. Addition and concepts of complex PTSD and prolonged grief disorder were explained earlier in this paper. Adjustment disorder is defined on the basis of the core feature of preoccupation (rather than only response) with a life stressor or its consequences, which indicates a conceptual change in ICD-11 and classified in "factors influencing health status or contact with health services." ${ }^{27}$

\section{Dissociative Disorders}

The term "conversion" in ICD-10 is eliminated from the group title 68. International classification of diseases-11 dissociative neurological symptom disorder is presented as a single disorder with 12 subtypes, which is conceptually consistent with ICD-10 dissociative disorders of movement and sensation. Dissociative fugue is not counted as a separate disorder but kept as a qualifier for dissociative amnesia. The ICD-10 possession trance disorder is separated into trance disorder (typically involves the repetition of a small repertoire of simpler behaviors) and possession trance disorder (a greater range of more complex behaviors exhibited). Dissociative identity disorder in ICD-11 corresponds to the concept of ICD-10 multiple personality disorder. Introduction of partial dissociative identity disorder covers several cases classified as unspecified in ICD-10. Depersonalization and derealization disorder is moved to the dissociative disorders group in the ICD-11 from other neurotic disorders group in the ICD-10.

\section{Feeding and Eating Disorders}

Due to life-span approach, feeding and eating disorders of childhood have been included as these are interconnected and could appear in individuals across a broader range of ages. ${ }^{41}$ Other than the addition of ARFID and binge eating disorder, conceptual upgradation is there in eating disorders according to recent researches. In the diagnosis anorexia nervosa, requirement for the presence of a widespread endocrine disorder has been eliminated; the threshold for low body weight in ICD-11 is raised from $17.5 \mathrm{~kg} / \mathrm{m}^{2}$ to $18 \mathrm{~kg} / \mathrm{m}^{2}$. Bulimia nervosa can be diagnosed regardless of the current weight until the body mass index is not very low and even if there is only "subjective" binges in the absence of "objective" binges. $^{29}$

\section{Elimination Disorders}

The term "non-organic" is removed from the ICD-11.

\section{Disorders of Bodily Distress and Bodily Experience}

The group includes two disorders: bodily distress disorder (represents ICD-10 concepts of somatoform disorders and neurasthenia) and body integrity dysphoria (mentioned earlier). While describing bodily distress disorder, more importance is given on excessive attention and preoccupation given on somatic complaints rather than the mere absence of medical explanations for bothersome symptoms. ${ }^{41}$ Qualifiers are (mild, moderate, or severe) in concordance with dysfunctioning.

\section{Disorders Due to Substance Use and Addictive Behaviors}

The major conceptual change entertained in substance use disorders is the addition of "single episode of harmful substance use," which provides an opportunity for early intervention and prevention of escalation of use or harm, whereas the diagnoses of "harmful pattern" of substance use and substance dependence suggest the need for intensive interventions. Importantly, the so-called behavioral addictions naming gambling disorder (pathological gambling in ICD-10) and gaming disorder ${ }^{32}$ were introduced in this grouping. Recent evidence points toward similar neurobiology, especially activation and neuro-adaptation within the reward and motivation circuits, ${ }^{42}$ and important phenomenological similarities like a change from "impulsive to compulsive" use.

\section{Impulse Control Disorders}

The group includes kleptomania and pyromania. Significant changes are the introduction of intermittent explosive disorder and compulsive sexual behavior disorder, ${ }^{34,43}$ which reclassifies ICD-10 excessive sexual drive.

\section{Disruptive Behavior and Dissocial Disorders}

The group includes oppositional defiant disorder and conductdissocial disorder. The new term was put for better reflecting the full range of severity of behaviors. Such disorders can be diagnosed across the life span; however, a qualifier has been added based on prognosis (earlier onset indicates more severe pathology and a more miserable course). The new addition of disruptive mood dysregulation disorder in DSM-5 invited much criticism and ICD-11 tactfully avoids that by adding of another qualifier "with chronic irritability and anger," which is based on the evidence that its presence significantly increases the risk for subsequent depression and anxiety. ${ }^{44}$

International classification of diseases-11 conduct disorder consolidates the three separate conduct disorder diagnoses (i.e., confined to the family context, unsocialized, socialized) classified in ICD-10, as psychosocial risk factors act simultaneously rather than separately. ${ }^{45}$

\section{Personality Disorders}

The crucial change entertained in ICD-11 was the addendum of qualifiers-mild, moderate, or severe-based on the pervasiveness of disturbances in the functioning of self, interpersonal dysfunction; severity and chronicity of emotional, cognitive, and behavioral manifestations; and the extent of psychosocial impairment. Practically as per ICD-10 classification, it had been noted that emotionally unstable personality disorder, borderline type, and dissocial personality disorder had been most frequently diagnosed condition in publicly available databases. ${ }^{46}$ Five trait domains were included: negative affectivity, detachment, dissociality, disinhibition, and anankastia. A new category naming "personality difficulty" actually refers to those individuals where deviant traits are present not up to the severity amounting to be labeled as a disorder. ${ }^{10}$

\section{Paraphilic Disorders}

Paraphilic disorder is the contemporary terminology more frequently used in research and clinical contexts and so is assigned to the group name than ICD-10 grouping of disorders of sexual preference. More focus was put on the fact that behaviors were acted on non-consenting others; resultantly, private behaviors, such as sadomasochism, fetishism, and fetishistic transvestism, have been eliminated as those found to be not relevant to public health. However, disorders like asphyxophilia, although being a solitary 
private behavior, is kept in this group for an obvious reason under "other paraphilic disorder." 46

\section{Factitious Disorders}

The new grouping of factitious disorders includes factitious disorder imposed on the self and factitious disorder imposed on another. Malingering is not classified as a mental, behavioral, or neurodevelopmental disorder but appears in the chapter on "factors influencing health status or contact with health services."

\section{Neurocognitive Disorders}

The group includes delirium, mild neurocognitive disorder (called mild cognitive disorder in ICD-10), amnestic disorder, and dementia. The syndromal characteristics of dementia are classified in the chapter on mental, behavioral, and neurodevelopmental disorders, whereas the underlying etiologies are classified in the chapter on diseases of the nervous system or other sections as appropriate. Dementia may be classified as mild, moderate, or severe. ${ }^{47}$

\section{Conclusion}

For a classification of mental disorders, development of the ICD-11 has been the most global, multilingual, multidisciplinary, and participative revision process by far. The ICD-11 mortality and morbidity statistics would be used by all member states for health statistics, as well as CDDG would be used in clinical settings, subjected to be modified according to individual country's law, policies, and health structures. The major innovations like the incorporation of the life-span approach, integration of the dimensional approach, the inclusion of culturerelated information, inclination toward the biological approach, along with effort for destigmatization obviously strengthen its structure. Being the successor of DSM-5 in a time line, it probably could avoid few loopholes and criticisms arose for DSM-5; however, in most instances, these two classificatory systems have been tuned in the same frequency. So if a unified classification system is sought to be used worldwide, they probably would not be irrelevant.

\section{References}

1. World Health Organization. ICD-11 for mortality and morbidity statistics (ICD-11 MMS) 2018 version. https://icd.who.int/browse11/ I-m/en.

2. First MB, Rebello TJ, Keeley JW, et al. Do mental health professionals use diagnostic classifications the way we think they do? A global survey. World Psychiatry 2018;17(2):187-195. DOI: 10.1002/wps.20525.

3. International Advisory Group for the Revision of ICD-10 Mental and Behavioral Disorders. A conceptual framework for the revision of the ICD-10 classification of mental and behavioral disorders. World Psychiatry 2011;10:86-92. DOI: 10.1002/j.2051-5545.2011.tb00022.x.

4. Bank W. World Bank country and lending groups. World Bank Data Help Desk Washington, DC; 2017.

5. First MB. The importance of developmental field trials in the revision of psychiatric classifications. Lancet Psychiatry 2016;3(6):579-584. DOI: 10.1016/S2215-0366(16)00048-1.

6. Keeley JW, Reed GM, Roberts MC, et al. Developing a science of clinical utility in diagnostic classification systems: Field study strategies for ICD-11 mental and behavioral disorders. Am Psychol 2016;71(1):3. DOI: $10.1037 / \mathrm{a} 0039972$.

7. Reed GM, Keeley JW, Rebello TJ, et al. Clinical utility of ICD-11 diagnostic guidelines for high-burden mental disorders: results from mental health settings in 13 countries. World Psychiatry 2018;17(3):306-315. DOI: 10.1002/wps.20581.
8. Clark LA, Cuthbert B, Lewis-Fernández R, et al. Three approaches to understanding and classifying mental disorder: ICD-11, DSM-5, and the National Institute of Mental Health's Research Domain Criteria (RDoC). Psychol Sci Public Interest 2017;18(2):72-145. DOI: 10.1177/1529100617727266.

9. Markon KE. Modeling psychopathology structure: a symptom-level analysis of axis I and II disorders. Psychol Med 2010;40(2):273-288. DOI: 10.1017/S0033291709990183.

10. Reed GM. Progress in developing a classification of personality disorders for ICD-11. World Psychiatry 2018;17(2):227-229. DOI: 10.1002/wps.20533.

11. Roberts MC, Reed GM, Medina-Mora ME, et al. A global clinicians' map of mental disorders to improve ICD-11: analysing meta-structure to enhance clinical utility. Int Rev Psychiatry 2012;24(6):578-590. DOI: 10.3109/09540261.2012.736368.

12. Reed GM, Roberts MC, Keeley J, et al. Mental health professionals' natural taxonomies of mental disorders: implications for the clinical utility of the ICD-11 and the DSM-5. J Clin Psychol 2013;69(12): 1191-1212. DOI: 10.1002/jclp.22031.

13. Reed GM, Drescher J, Krueger RB, et al. Disorders related to sexuality and gender identity in the ICD-11: revising the ICD-10 classification based on current scientific evidence, best clinical practices, and human rights considerations. World Psychiatry 2016;15(3):205-221. DOI: 10.1002/wps.20354.

14. Chakrabarti SU. Psychotic and catatonic presentations in bipolar and depressive disorders. World Psychiatry 2012;11(Suppl 1): 59-64.

15. Vieta E, Suppes T. Bipolar II disorder: arguments for and against a distinct diagnostic entity. Bipolar Disord 2008;10(1p2):163-178. DOI: 10.1111/j.1399-5618.2007.00561.x.

16. Solé B, Martínez-Arán A, Torrent $C$, et al. Are bipolar II patients cognitively impaired? A systematic review. Psychol Med 2011;41(9):1791-1803. DOI: 10.1017/S0033291711000018.

17. Wang Z, Li Z, Chen J, et al. Association of BDNF gene polymorphism with bipolar disorders in Han Chinese population. Genes Brain Behav 2012;11(5):524-528. DOI: 10.1111/j.1601-183X.2012.00797.x.

18. Chou Y-H, Wang S-J, Lin C-L, et al. Decreased brain serotonin transporter binding in the euthymic state of bipolar I but not bipolar II disorder: a SPECT study. Bipolar Disord 2010;12(3):312-318. DOI: 10.1111/j.1399-5618.2010.00800.x.

19. Ha TH, Ha K, Kim JH, et al. Regional brain gray matter abnormalities in patients with bipolar II disorder: a comparison study with bipolar I patients and healthy controls. Neurosci Lett 2009;456(1):44-48. DOI: 10.1016/j.neulet.2009.03.077.

20. Stein DJ, Kogan CS, Atmaca M, et al. The classification of obsessivecompulsive and related disorders in the ICD-11. J Affect Disord 2016;190:663-674. DOI: 10.1016/j.jad.2015.10.061.

21. Veale $D$, Matsunaga $H$. Body dysmorphic disorder and olfactory reference disorder: proposals for ICD-11. Rev Bras Psiquiatr 2014;36(Suppl 1):14-20. DOI: 10.1590/1516-4446-2013-1238.

22. Suzuki K, Takei N, Iwata $\mathrm{Y}$, et al. Do olfactory reference syndrome and jikoshu-kyofu (a subtype of taijin-kyofu) share a common entity? Acta Psychiatr Scand 2004;109:150-155. DOI: 10.1046/j.16000447.2003.00195.x.

23. Mataix-Cols D, Fernández de la Cruz L. Hoarding disorder has finally arrived, but many challenges lie ahead. World Psychiatry 2018;17(2):224-225. DOI: 10.1002/wps.20531.

24. Timpano KR, Exner C, Glaesmer H, et al. The epidemiology of the proposed DSM-5 hoarding disorder: exploration of the acquisition specifier, associated features, and distress. J Clin Psychiatry 2011. DOI: 10.4088/JCP.10m06380.

25. Fontanelle LF, Grant JE. Hoarding disorder: a new diagnostic category in ICD-11. Rev Bras Psiquiatr 2014;36(Suppl 1):28-39. DOI: 10.1590/1516-4446-2013-1269.

26. Grant JE, Stein DJ. Body-focused repetitive behavior disorders in ICD-11. Braz J Psychiatry 2014;36:59-64. DOI: 10.1590/1516-44462013-1228. 
27. Maercker A, Brewin CR, Bryant RA, et al. Diagnosis and classification of disorders specifically associated with stress: proposals for ICD-11. World Psychiatry 2013;12(3):198-206. DOI: 10.1002/wps. 20057.

28. Shear K, Frank E, Houck PR, et al. Treatment of complicated grief: a randomized controlled trial. JAMA 2005;293(21):2601-2608. DOI: 10.1001/jama.293.21.2601.

29. Uher R, Rutter M. Classification of feeding and eating disorders: review of evidence and proposals for ICD-11. World Psychiatry 2012;11(2):80-92. DOI: 10.1016/j.wpsyc.2012.05.005.

30. Al-Adawi S, Bax B, Bryant-Waugh R, et al. Revision of ICD-status update on feeding and eating disorders. Adv Eat Disord 2013;1(1): 10-20. DOI: 10.1080/21662630.2013.742971.

31. First MB, Fisher CE. Body integrity identity disorder: the persistent desire to acquire a physical disability. Psychopathology 2012;45(1): 3-14. DOI: 10.1159/000330503.

32. Saunders JB, Hao W, Long J, et al. Gaming disorder: its delineation as an important condition for diagnosis, management, and prevention. J Behav Addict 2017:6:271-279. DOI: 10.1556/2006.6.2017.039.

33. Kraus SW, Krueger RB, Briken $\mathrm{P}$, et al. Compulsive sexual behavior disorder in the ICD-11. World Psychiatry 2018;17(1):109-110. DOI: 10.1002/wps.20499.

34. Coccaro EF. Intermittent explosive disorder as a disorder of impulsive aggression for DSM-5. Am J Psychiatry 2012;169(6):577-588. DOI: 10.1176/appi.ajp.2012.11081259.

35. Figueira ML, Dias VV. Postpartum depression and premenstrual dysphoric disorder: options for ICD-11. World Psychiatry 2012;11(Suppl 1):73-76.

36. Tassé MJ, Balboni G, Navas P, et al. Developing behavioral indicators for intellectual functioning and adaptive behavior for ICD-11 disorders of intellectual development. J Intellect Disabil Res 2019;63(5):386-407. DOI: 10.1111/jir.12582.

37. Gaebel W. Status of psychotic disorders in ICD-11. US: Oxford University Press; 2012.
38. McGlinchey JB, Zimmerman M, Young D, et al. Diagnosing major depressive disorder VIII: are some symptoms better than others? J Nerv Ment Dis 2006;194(10):785-790. DOI: 10.1097/01. nmd.0000240222.75201.aa.

39. Paykel E, Andrade LH, Njenga F, et al. Changes needed in the classification of depressive disorders: options for ICD-11. World Psychiatry 2012;11(Suppl 1):37-42.

40. Kogan CS, Stein DJ, Maj M, et al. The classification of anxiety and fear-related disorders in the ICD-11. Depress Anxiety 2016;33(12): 1141-1154. DOI: 10.1002/da.22530.

41. Gureje O, Reed GM. Bodily distress disorder in ICD-11: problems and prospects. World Psychiatry 2016;15:291-292. DOI: 10.1002/ wps.20353.

42. Fauth-Bühler M, Mann K, Potenza MN. Pathological gambling: a review of the neurobiological evidence relevant for its classification as an addictive disorder. Addict Biol 2017;22(4):885-897. DOI: 10.1111/ adb.12378.

43. McLaughlin KA, Green JG, Hwang I, et al. Intermittent explosive disorder in the national comorbidity survey replication adolescent supplement. Arch Gen Psychiatry 2012;69(11):1131-1139. DOI: 10.1001/ archgenpsychiatry.2012.592.

44. Lochman JE, Evans SC, Burke JD, et al. An empirically based alternative to DSM-5's disruptive mood dysregulation disorder for ICD-11. World Psychiatry 2015;14(1):30-33. DOI: 10.1002/wps.20176.

45. Evans SC, Burke JD, Roberts MC, et al. Irritability in child and adolescent psychopathology: an integrative review for ICD-11. Clin Psychol Rev 2017;53:29-45. DOI: 10.1016/j.cpr.2017.01.004.

46. Krueger RB, Reed GM, First MB, et al. Proposals for paraphilic disorders in the international classification of diseases and related health problems, eleventh revision (ICD-11). Arch Sex Behav 2017;46: 1529-1545. DOI: 10.1007/s10508-017-0944-2.

47. Gaebel W, Jessen F, Kanba S. Neurocognitive disorders in ICD-11: the debate and its outcome. World Psychiatry 2018;17:229-230. DOI: 10.1002/wps.20534. 